\title{
Exploring the acceptance of telehealth within palliative care: A self-determination theory perspective
}

\author{
Joseph Keenan ${ }^{1} \cdot$ Rachel Rahman $^{2} \cdot$ Joanne Hudson $^{3}$
}

Received: 19 January 2021 / Accepted: 18 February 2021 / Published online: 4 March 2021

(c) The Author(s) 2021

\begin{abstract}
The aim of this paper was to explore potential divergence and convergence in relation to health care professionals' and patients' acceptability of the use of telehealth within palliative care provision through the lens of Self-Determination Theory. The research utilized a deductive qualitative approach utilizing semi-structured interviews to explore divergence and convergence between health care professionals' preconceptions of the use of telehealth in palliative care and the lived experiences of patients accessing support in this manner. Semi-structured interviews were conducted with both professionals and patients to explore whether the barriers and benefits of telehealth perceived by professionals corresponded to the patient's lived experience of utilizing the technology in their palliative care. Interviews were analyzed using a deductive thematic analysis. Professionals and patients identified that the use of telehealth could satisfy the need for autonomy, however this manifested in different ways. Greater divergence was apparent between patient and professional perceptions about how telehealth could satisfy the need for relatedness and competence needs. The findings of this paper highlight how professionals preconceived concerns about the use of telehealth in relation to providing supportive palliative care may not be realized when exploring the experiences of patients accessing services through this medium. This paper highlights the important role of psychological need satisfaction when considering acceptability of telehealth, and motivation to engage in the implementation of technologically driven health services.
\end{abstract}

Keywords Telehealth $\cdot$ Palliative Care $\cdot$ Self-Determination $\cdot$ Thematic Analysis

\section{Background}

Many countries are experiencing challenges in health care delivery, including the increased pressures of an aging population, staff shortages and the need to deliver services closer to patient homes [1]. Palliative care delivered in rural areas represents one area of health care provision faced with challenges to service delivery [2]. Numerous policy documents $[3,4]$ identify telehealth as a potential way of overcoming some of these challenges. Through widening opportunities for community-based care, improving access

Joseph Keenan

j.keenan@mmu.ac.uk

1 Department of Psychology, Manchester Metropolitan University, Manchester, England, UK

2 Department of Psychology, Aberystwyth University, Aberystwyth, Wales, UK

3 College of Engineering, Swansea University, Swansea, Wales, UK to services for rural and remote patients, and decreasing the travel time of patients thus contributing to their quality of life, without a corresponding increase in travel time for professionals, thus ensuring a more economically efficient service.

The palliative care experience is highly emotive for both patients and professionals, and high quality relationships are paramount. Consequentially, a greater understanding of the tensions of using telehealth to facilitate relationships between patients and professionals during palliative care is required. Pilot implementation studies have consistently reported positive patient perceptions and acceptability of technology in palliative care [5-7] and occasionally report patient frustrations that professionals fail to make more use of the technology available to them [8]. Research has highlighted how the acceptance of telehealth by professionals are central to the implementation of telehealth within traditional methods of health care delivery [9] and professionals remain an important gatekeeper to the services that are made available to their patients. 
From a professional perspective, research suggests that they may remain more sceptical of telehealth in comparison to their patient counterparts. This can be theoretically explained through Normalization Process Theory (NPT) in relation to interactional workability, relational integration, skill-set workability, and contextual integration $[10,11]$. The preconceived negative perceptions of professionals towards telehealth may potentially limit a service being offered, resulting in patients not getting to contribute their views on new methods of service delivery.

It appears therefore, that the perceived barriers of professionals towards telehealth implementation may be a divide between patients and policy makers achieving what they perceive to be the solution to their unmet needs. However, whilst research has previously considered the practical barriers and benefits of adoption there is limited research that has explored the deeper psychological reasons that individuals may hold differing perspectives.

There is therefore a need to consider theoretically, where there may be agreement and disagreements in the underlying psychological values and views of stakeholders in order to enable solutions or research to unlock the potential of telehealth for relevant groups where appropriate. SelfDetermination Theory [12] is a theory of motivation that considers how fulfilment of psychological needs determines our motivations to engage in certain behaviours. The theory proposes three inherent psychological needs, autonomy, competence, and relatedness. Autonomy relates to a need to feel self-determined and volitional in one's decisions and behaviours. Competence relates to the need to feel able to affect outcomes of one's behaviours and relatedness represents the need to feel supported and accepted within one's environment. According to SDT, satisfaction of the psychological needs is associated with increased likelihood of engaging in a behaviour [13], increased persistence and improved well-being, whilst a lack of satisfaction or active thwarting of psychological needs can result in a lack of motivation to engage and poorer psychological wellbeing.

Applying SDT to the implementation of telehealth therefore provides a novel opportunity to consider how professional and patients narratives of the barriers and benefit of telehealth represents potential satisfaction or dissatisfaction of underlying psychological needs. Identifying these may provide insight into the latent psychological barriers and facilitators to telehealth implementation thus better informing future implementation strategies. The present research hopes to contribute a novel perspective to the debate of why telehealth has not been adopted on a larger scale despite evidence suggesting that its incorporation in to health care delivery can result in positive psychological and clinical outcomes for patients and other service users [5]. To achieve this, the current paper considers data from differing stakeholders exploring the use of telehealth in palliative care from the perspectives of professionals and patients respectively.
The research therefore aims to address the research question: What are the convergent and divergent views held by professionals and patients towards the implementation of telehealth in palliative care in relation to the principles of Self-Determination Theory? The question was framed upon cross-examination and reanalysing of data in two pilot exploratory studies. The studies looked at the two stakeholder groups independently and identified potential divergence and convergence in relation to the role of SDT underpinning the acceptability of telehealth within palliative care. This warranted further secondary analysis and pooling of the data from each stakeholder group.

\section{Method}

\subsection{Design}

This paper utilizes a qualitative design to gain in-depth insight regarding the preconceived barriers and benefits of telehealth use in palliative care held by professionals in relation to their patients' lived experiences of using telehealth to access psychosocial support while receiving palliative care.

To explore professional perceptions semi-structured interviews were conducted at one time point ahead of the introduction of a telehealth service. Due to the focus of the interviews with health care professionals being related to their perceptions of telehealth interviews were not conducted at subsequent time points. Patient data was collected through a series of semi-structured interviews conducted at three monthly interviews following the introduction of the telehealth service.

\subsection{Participants}

A sample of eight professionals and three palliative care patients (each with 3 data points) were included in this study and provided a total of $12 \mathrm{~h}$ of data.

\subsection{Health care professionals}

Professionals were identified through their employment with a rurally based voluntary palliative care hospice at home service and those working within a specialist palliative care team at the local general hospital.

Only professionals responsible for a caseload of patients, providing either clinical or social care to palliative care patients were approached. Exclusion criteria included professionals who did not actively contribute to either clinical or social care provision of patients accessing the palliative care service.

A demographic overview of the professionals included in this study can be found in Table 1 . 
Table 1 Demographic information of health care professionals included in the sample

\begin{tabular}{llllll}
\hline Pseudonym & Age & Job Role & $\begin{array}{l}\text { Palliative care experi- } \\
\text { ence (years) }\end{array}$ & $\begin{array}{l}\text { Prior experience } \\
\text { using telehealth }\end{array}$ & Gender \\
\hline Hannah & 58 & GP & 28 & Yes & Female \\
Sian & 42 & District nurse & 15 & Yes & Female \\
Lisa & 43 & District nurse & 17 & No & Female \\
Stephanie & 38 & Specialist nurse & 13 & Yes & Female \\
Eva & 54 & Consultant & 27 & No & Female \\
Georgia & 38 & Case worker & 7 & No & Female \\
Grace & 57 & Psychotherapist & 23 & Yes & Female \\
Ellie & 48 & Consultant & 21 & Yes & Female
\end{tabular}

\subsection{Patients}

Members of the palliative care multidisciplinary team initially approached patients from their caseloads. Inclusion criteria for the study consisted of patients who were recipients of specialist palliative care from the multidisciplinary team and who lived in a rural location. In the case of this study, rural was determined as living at least a 40-min drive from the specialist palliative care unit at the local general hospital (not a specialist tertiary centre). Exclusion criteria consisted of patients who were under the age of eighteen and those who did not possess the capacity to provide informed consent to take part in the study. Participants who did not possess a working internet connection also had to be excluded as this was required to facilitate the telehealth service.

Of the participants recruited to the study, each of the participants were receiving palliative care following a diagnosis of terminal cancer. Two of the participants in a private residence alone, while the remaining participant resided in a residential nursing home. A demographic overview of the participating patients and their associated pseudonyms can be found in Table 2 .

\section{Procedure}

\subsection{Health care professionals}

Professionals were provided with a detailed information sheet providing an overview of the research process. It was emphasised that involvement in the study was voluntary and that any views expressed would be anonymized. Following

Table 2 Demographic information of patients included in the sample

\begin{tabular}{llll}
\hline Pseudonym & Age & Gender & Primary diagnosis \\
\hline Patricia & 48 & Female & Breast Cancer \\
Caroline & 63 & Female & Lung Cancer \\
Judith & 72 & Female & Bone Cancer \\
\hline
\end{tabular}

informed consent being provided, professionals responded to questions concerning their perceptions of telehealth use in palliative care in a semi-structured interview. The interview itself consisted of inter-related sections based upon Spradley's model of building interviewer-interviewee rapport [14]. Driven by an extensive literature review, the interviewer asked questions pertaining to the multifunctional role of professionals in palliative care provision, their understanding of the concept of telehealth, their perceived practical and psychological barriers and benefits of implementation in relation to existing service provision.

\subsection{Patients}

Patients who expressed an interest in the study following a conversation with members of their health care team received a participant information sheet with a reply slip attached. Participants were asked to complete the reply slip and return to the research team who were then able to contact them directly to address any questions and arrange a convenient time for an interview. This was to ensure that no patient details were provided to the research team ahead of consent being provided. For convenience, interviews were conducted in patients' homes.

The subsequent visit to the participant's home involved a general introduction and a face-to-face demonstration of the equipment. Polycom RealPresence $\odot$ software was installed on laptop computers, which were provided to each participant for the duration of the study. The Polycom RealPresence software accessed a remote VPN that enabled the remote laptops to access the secure NHS network.

Following demonstration and trial run connections, the participant received written systematic instructions of how to connect to reinforce the visual demonstration for future reference throughout the duration of the project.

Participants accessed psychosocial support from a qualified therapist who was part of the palliative care team via the telehealth system on average once every ten days (Range: 8-12) and lasted up to one hour. The support 
received focused mainly on the provision of psychosocial support on a one to one basis, which on occasions utilised techniques such as art therapy. Sessions were arranged through discussion between the professional and patient and the use of an appointment system in the same manner as face-to-face appointments.

Interviews were conducted with patients on three occasions at monthly intervals following the introduction of the technology. Additional prompts were added to the second and third interview schedule to investigate whether patients had made sense of their experience differently in comparison to their prior interviews. Interviews were audio recorded for the purpose of transcription. Interviews ranged in length (from 21 to $79 \mathrm{~min}$ ) determined by the health of the patient.

\subsection{Data analysis}

The analysis utilized a deductive thematic analysis using the basic psychological needs of Self-Determination Theory [12] as a guiding theoretical framework. A deductive approach is useful if the general aim of thematic analysis is to test a previous theory in a different situation, or compare categories at different periods $[15,16]$. In the case of this paper, the deductive approach was utilized to explore the role of psychological need satisfaction in the context of professional preconceptions of telehealth and the lived experience of palliative care patients using telehealth. The analytical technique followed the guidelines of [17].

Figure 1 provides an overview of the analytical process for both stakeholder groups. With reference to interviews conducted with professionals, interviews were treated as a whole data set and were coded as such. In order to explore the multiple time-points interviews conducted with patients a slightly modified analytical approach was taken. Coding was initially conducted at time-point level for each individual patient. Once completed, convergence and divergence were explored between time-points for each participant. The generated codes were then collated into themes based upon the principles of SDT for each individual patient. Once completed, a cross-case comparison of the patients was conducted exploring the convergence and divergence across cases.

The themes identified for each stakeholder group were then pooled together to explore the convergence and divergence of themes across the two stakeholder groups.

\subsection{Ethical Considerations}

Ethical approval was gained from the local NHS Research Ethics Committee and NHS Research and Development. Participants provided written informed consent prior to participation in the research.

For ethical requirements, the researcher was accompanied to interviews with patients by the professional responsible for providing support to the patient. During the interview the professional was situated in a different room but could be called on should any patients become distressed during the interview. A clear explanation that telehealth would be removed after 3 months was provided; however, where the professionals felt patients were particularly benefitting and in need of the service, agreements were made to enable the service to keep the laptops for continuation of the service.

\subsection{Findings}

Using principles of Self-Determination Theory [12], three themes were identified autonomy, competence, and relatedness. Within each theme the convergent and divergent

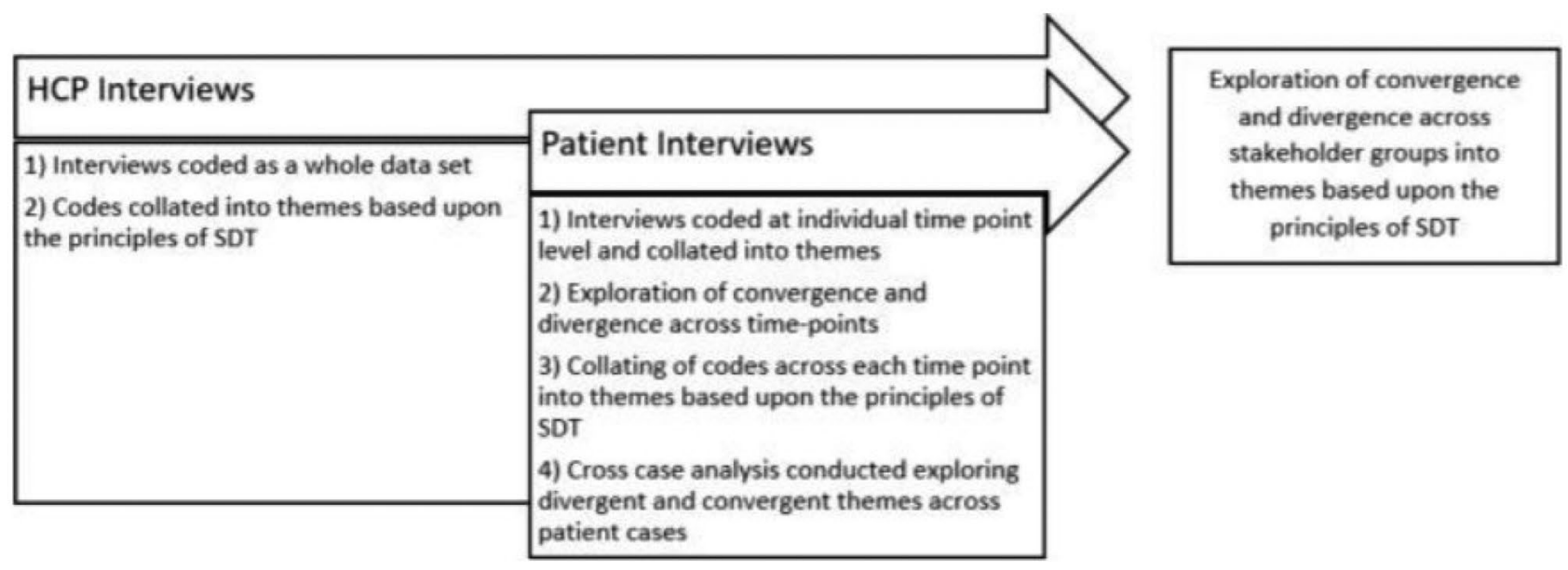

Fig. 1 Visual overview of analytic process 
perceptions of professionals and patients was explored in relation to either the satisfaction or thwarting of their basic psychological needs in the context of using telehealth in palliative care.

\subsection{Autonomy}

Within the theme of Autonomy, both professionals and patients suggest that telehealth facilitated an autonomy supportive environment. However, professionals discussed autonomy support from a more condition-centred perspective in comparison to patients who reflected on autonomy from a more holistic life-centred perspective. In contrast, professionals and patients demonstrated divergent attitudes in relation to telehealth being able to create an environment that supported the needs for competence and relatedness.

Both professionals and patients suggested that telehealth could play a role in empowering patients and increasing their sense of autonomy; however, there was an evident difference in how this sense of autonomy satisfaction manifested itself. Professionals discussed the use of telehealth from a condition-centred perspective, whereas patients considered the need for autonomy in a more holistic manner.

Professional perceptions of telehealth often centred on discussion of telemonitoring systems as opposed to other forms of telehealth such as videoconferencing or text message reminder services. This is interesting in the context of telehealth adoption where professionals may not be considering the broad range of applications that could be of benefit to their service. In this context professionals saw telehealth as an opportunity for patients to selfmanage their illness and symptoms via feedback received from telemonitoring systems. This in turn was perceived to facilitate patient autonomy through an improved ability to understand their conditions and the need for selfmanagement techniques.

"I think there is also an element of importance in the terms of the individual feeling they have a sense of self management because if they can see what's happening in terms of how they behave and how it affects their symptoms it will help them as well" Georgia (Social worker), 255.

Patients also identified how telehealth could create a more autonomy supportive environment and improve opportunities to understand and learn more about their condition through increased confidence to engage in questions and discussion with professionals. In the following extract, Patricia discusses how she experienced feeling less daunted when talking to a professional and feeling increased confidence to ask questions during interactions.
"I think sometimes when you go face-to-face, especially with some of the big consultants, it's kind of a bit daunting or a bit off putting or they're kind of a bit, like, okay I've got so much time there's a whole waiting room of people out there. Whereas if you're on telehealth maybe it would give you a bit more confidence to say, oh wait a minute I need to just ask you this or hang on I need to ask you this. And just give you that bit more confidence because you're not actually there with them,... you're in their space aren't you, whereas at home you're on your own grounds, so you're kind of more confident anyway" Patricia, Time point 2, 202.

This suggests that telehealth facilitates an autonomy supportive environment that encourages patients to feel empowered to interact more fully with healthcare professionals. This interpretation mirrors the description of an autonomy supported environment by Reeve et al. [18] as environments that inclusive of opportunities for participation and choice, acknowledgement of negative feelings associated with engagement in difficult tasks, and minimization of external controls [18]. The perceived remoteness and confidence of being on familiar territory offered increased confidence to ask questions and seek support that in their eyes would have been more challenging in a face-to-face consultation.

Patients also described their experience of autonomy satisfaction in a more life-centred manner, focusing on the liberation that telehealth consultation provided through the reduction in travel burden to attend appointments. An example of this liberation was discussed through Patricia's reflection of how using telehealth to access support provided her with the freedom to plan the rest of her day.

"So, to do a session yesterday was good because I think we went on at half-nine. So, I sort of got up, had breakfast, did a few things and then did that. Then the rest of the day - as soon as you sign off the rest of the day is your own. But if I'd gone to [location of hospital] it's pretty much the whole day really because, it doesn't take you the whole day to get there obviously, but because I get tired when I do that sort of thing then that would be me finished for the rest day. That would be what I'd do for that day. Whereas sitting here in your own lounge on a computer is not as tiring by any stretch of the imagination, so it kind of leaves you more time to do other things."

Patricia - Time point $1-72$.

This liberation enabled patients to divert their energy into more enjoyable or fulfilling activities and demonstrated that engagement with technology was not just about managing their condition but reopened the opportunity to feel volitional about other more meaningful areas of their lives. 
The above extracts highlight that both professionals and patients perceived that the use of telehealth could help empower patients and provide an environment in which the need for autonomy was supported. However, when exploring how they made sense of this environment a divergence emerges in relation to how stakeholders felt this would be achieved. Professionals made sense of empowerment from a condition-centred perspective, through discussion of selfmanagement of symptoms. In contrast patients placed more importance on a more life-centred and holistic concept of autonomy.

This finding may challenge how successfully professionals have fully embraced holistic patient-centred care as opposed to traditional biomedical models where the clinical management of the condition is considered more prominently than the broader environment within which the patient is experiencing their health. Thus, whilst professionals maintain that face-to-face care is superior to that of telehealth consultations, it may be that professionals are failing to consider the tension between a face to face consultation and the corresponding pressures of patients to attend. The additional burden of travel, fatigue and organization of their daily activities around appointments detracts from the benefits of a face-to-face meeting and leaves patients in a position where they fail to engage fully in consultations.

The additional reflection by patients that the remoteness offered by technology and the home setting of consultations was also interesting and raises novel perspectives on how certain patients may experience interactions with professionals and have implications for health care delivery more broadly that simply those in rural locations.

\subsection{Competence}

The competence of providers and patients to manage technology as part of routine care was discussed by both groups. Interestingly, both professionals and patients use age as a moderating discussion point in reference to competence of both service providers and users. Both groups consider the use of telehealth to be more acceptable to younger patients due to them being more able to develop the required competency for use. However, when discussing their experience of using the technology, the patients within the sample suggested a sense of mastery and few issues relating to the usability of the equipment, divergent to both their own preconceptions of older users' competence, and the perceptions of professionals.

In all of the interviews conducted with professionals age was perceived to be a potential barrier to the successful adoption of telehealth within palliative care delivery.
"I do think that the young would be far more accepting of it and would probably find it cool. They may also be able to express themselves better because they already express themselves to a screen with Facebook or Twitter. I think they would have no issues whatsoever. My concerns are that... a large proportion of my patients are elderly and they would find it quite odd" Eva (Specialist nurse), 75.

Professionals had made pre-existing judgments about how telehealth would potentially thwart the satisfaction of patient needs for competence and had made decisions about how older adults in their care would cope with technology adoption. As such, professionals perceived that telehealth could be implemented more easily to support their relationships with younger patients. A wealth of literature supports the interpretation that age plays a moderating role in the acceptance of technology and how this relates to supplementing relationships between professionals and the patients. The Unified Theory of the Acceptance and use of Technology (UTAUT) [19] suggests prior experience of using technology is a facilitating factor in the decision to accept technology. This perception links to previous research conducted by Radhakrishnan et al. [20] who investigated telehealth use with patients suffering from heart failure. When analysing the use of the equipment, they reported a prevalence of elderly heart failure patients terminating the use of telehealth in their care program due to increasing levels of anxiety stemming from having to use the equipment [20]. With a high proportion of palliative care patients coming from the older generation, the age of patients could be perceived as a barrier by professionals towards the implementation of technology within palliative care due to their lack of familiarity using technology.

While patients largely agreed with professionals at baseline that younger individuals would find accessing support through telehealth easier, their own lived experience suggested a sense of mastery - demonstrated through their responses at different time-points using the equipment. The extract below taken from Patricia's first interview describes the initial stress encountered when using the telehealth system and reflecting on how this stress began to dissipate as the session unfolded.

"The first one when it happened when I got on, I did feel quite stressed. It was so hard and I thought this session is meant to be making me less stressed not more stressed. And I was like fit to pull my hair out and throw things through the window. But once I'd sort of talked about it with [health care professional] for a couple of minutes that kind of went out of my consciousness altogether then. I didn't kind of give it anymore thought after that really." 
When comparing the preceding extract to the one below, taken from an interview conducted two months later, Patricia reflected on her initial experiences and reflects upon the normalization of receiving psychosocial support in this way.

"I've got used to it [telehealth], I know more about it. You know, so it's not so alien if you like. Initially I didn't know what to expect at all. I didn't know how it would work. I mean, I'm not a technophobe, but I was like will I be able to work it and, you know, how is it sort of going to work sort of thing. And will it be the same as going in, is it going to take something away from that sort of thing. But I think as I'm going used to using it now, it's getting more comfortable and so I'm feeling that I'm getting the same amount of the sessions as I would if I was going into the hospital."

The concept of normalization and familiarity being key factors relating to the acceptance of technological innovation is not novel, with it forming the construct of numerous implementation models such as UTAUT [19] and Normalization Process Theory [21]. However, it appears that this may not be directly related to age with older adults in the study mastering the technology use and becoming increasingly competent at using the technology. It appears therefore that whilst both groups share the concern that older adults will not be suitable for telehealth use; in practice, the older adults in the study were willing to take steps to develop their competency and managed to master and find benefit in the telehealth system.

The divergence in the preconceived perceptions versus the lived experience may provide some explanation to the barriers of adoption. If professionals dismiss their older patients in their caseloads ability to use telehealth, there is a risk that older adults could be excluded from the opportunity to even consider telehealth use. The patient experience suggests that whilst telehealth may not be suitable for everyone. Many older adults, regardless of previous technological ability are potentially capable of developing mastery and satisfying the need for competence to utilize a simple videoconferencing system with suitable support in place. Particularly when this benefits him or her in other ways such as reduced travel and increased available time.

\subsection{Relatedness}

Divergence arose between professionals pre-conceived concerns of being able to develop trusting and continuous relationships with their patients and the experiences of the patients themselves. Professionals expressed concerns that the lack of proximity, absence of human touch, and remote nature of telehealth would prevent the development of trusting relationships, and minimize the ability of professionals to understand the emotional needs of their patients. In contrast, patient experiences of using telehealth to access psychosocial support demonstrated an environment where their needs for strong relationships and emotional understanding were satisfied.

One perception common across all interviews with professionals was the importance of human touch within this relationship. The lack of human touch was identified as a barrier for professionals as they feared that patients would consider the care provided using telehealth to be impersonal.

"I think the main barrier is that it's basically changing the way in which nurses work, erm nurses are caring people and nurses want to see people and I think that if you go and see someone who is unwell and put your hand on theirs, you know we touch people, it's what nurses do and that's part of the way that nurses care for people erm and I think to take that away and move to something that is a little more impersonal, might be a barrier to some nurses." Stephanie (specialist nurse), 396.

Some professionals, notably the nurses within the sample, reflected on perceptions that telehealth would ultimately change the way in which they provided care to their patients with concerns that a more remote form of care would create an unfamiliar environment to build supportive relationships.

The importance of human touch to the patientprofessional relationship has been researched in previous studies. Ludwig-Beymer [22] emphasised the importance stating that a "caring touch" can help connect and break down barriers, and express concern and interest, decrease anxiety, diminish pain and create a bond between people [22]. In relation to the implementation of telehealth, it has also been viewed as a potential barrier for both professionals and patients. Whitten et al. [8] discussed the notion that telehealth was viewed as impersonal, lacking in human touch, and that in a palliative care setting where the goal is to comfort patients and families, some clinicians viewed telehealth negatively [8]. Demiris et al. [23] discussed the impact on relationship between patients and professionals stating that when care provided remotely, human touch and other forms of nonverbal communication are lacking, and that, too, may affect the way in which both clinical understanding and personal relationships develop [23].

Professionals also perceived that the use of telehealth might present a potential barrier concerning providing emotional support to the patient.

"Good emotional support, I don't see that it can quite replace that element of this job. I mean palliative care nursing is specifically the BEING with a person it's the BEING there with a person that is absolutely key to the job I'm afraid. It's very well researched and documented. So you aren't there are you if you're video linking, so that's quite an important thing." Georgia (Social support worker), 215. 
Georgia suggests that in order to understand and support the emotional needs of palliative care patients, it is necessary for professionals to be present with the patient. This interpretation is supported by prior research which states that the dynamics observed in a video consultation can differ from a traditional face-to-face consultation, and interactional practices performed by professionals need to be adapted [24, 25]. Therefore, it is understandable that there will be on-going concerns regarding the doctor-patient relationship and rapport in relation to the delivery of clinical services through telehealth. Interestingly previous research has also demonstrated that facial expressions and emotions can be identified by professional through telehealth as effectively as face-toface communication [26].

However, despite professional perceptions that the remote nature of telehealth would lead to an impersonal service; the experiences of patients suggested that they were able to develop close connections and trusting relationships with the professionals providing their care.

Caroline suggested that she was able to feel relaxed and secure during her interaction with professionals during telehealth consultations. This was facilitated through a lack of distractions present in her home environment.

"Yeah, it like relaxes you, you're already relaxed.

You haven't had to do all the rushing and you're not in a strange place. You're not worried about other people hearing - well in my situation....but from my point of view, my experience of it, it's because there was nobody else in the home. Then you're totally relaxed that you can kind of say what you like, whereas if you're go to go into a building, you're not quite sure, are you sometimes, whether there are other people around." Caroline - Time point 3-66.

Caroline reflects on the benefit of a relaxing and familiar environment creating a sense of privacy in notable contrast to the preconceived 'unfamiliar' environment described by the professional. It is notable that for both groups that the environment of the other stakeholder is seen as unfamiliar and challenging. This provides novel insight into how despite professionals making decisions based on how they perceive their patients will respond, and attempting to adopt a patient centred approach, this may not always accurately reflect the true perceptions of their patients.

In contrast to professional perceptions, the importance of a physical presence for effective communication, patients also discussed how the solitude of connecting from their own home enabled a secure environment in which they felt comfortable to discuss personal feelings and needs.

"Well this is for me. I don't know about other people but for myself, like I said before, I feel that I'm on my own and I'm really just talking everything out, whereas if somebody like sat beside me, obviously you can pick up different senses and I don't think I'd be so open. So, I think yes, when I do talk on the computer, I'm very free, you just gabble. You just get it off your chest and it's good because normally, I'm not a talker really" Judith - Time point $1-56$.

For this patient the physical proximity of another individual seemingly limited her ability to talk freely. The telehealth equipment, whilst still being able to maintain a visual connection provided patients with the confidence to discuss their feelings without concerns about an immediate reaction from the professional. Thus, the patient experience suggests contrary to professional perceptions, there were potential benefits to the remoteness of the discussion. Patients were not suggesting that face-to-face meetings were unnecessary. However, it was apparent that for some patients that high quality communication was possible via telehealth and that this supported effective relationships with good relatedness support to be afforded and challenged the strong beliefs of the professionals that it was necessary to be face to face with patients to develop close relationships. In fact, it appears that the proximity and presence was valued more for supporting the psychological relatedness needs of the professional than that of the patient, despite the argument being proposed as patient centred.

\section{General Conclusion}

The current study aimed to explore whether the preconceived perceptions held by professionals regarding the use of telehealth in palliative care was convergent or divergent with the lived patient experience of using telehealth to access elements of their service from a SDT perspective. SDT [12] proposes that psychological needs influence behavioural regulation. As such understanding the different ways need satisfaction is discussed by patients and professionals provides a novel insight into the latent psychological reasons for barriers to routine telehealth use. The divergence identified between patient and professional perspectives on the capability of telehealth to establish an environment that satisfies basic psychological needs may allow us to understand why despite research suggesting telehealth can provide a supportive environment for patients; it has not been implemented more routinely within health care provision.

Given that service adoption often considered prior to patient engagement, the methodology employed in this study considered how professional views of telehealth ahead of service implementation corresponded or differed to the lived experience of the patient utilizing the service. This 
approach provides an interesting and novel opportunity to identify where preconceived barriers and benefits held by professionals, are either shared or challenged by the lived experience of the patient. Considering this within a theoretical needs-based framework also helps to inform how these shared or contradictory barriers may feed back into the motivations of relevant stakeholders for engaging in telehealth thus contributing a deeper psychological understanding of stakeholder perspectives.

Professionals consider telehealth to be a mechanism that could support patient autonomy satisfaction through education, feedback, and awareness of the condition. However, they appeared less aware of other forms of telehealth and the perceptions shared did not acknowledge the more holistic barriers of accessing support for patients, including stress caused by travel and the time and energy taken to attend appointments. Patients focused more on the ability of telehealth to satisfy autonomy by moving away from a condition-centred life and facilitating opportunities to engage in their usual everyday routines. This suggests that professionals try to assume the perspective of their patients when considering the appropriateness of technologies in their care and may not always consider the perspective of the patient, their holistic lives, and circumstances.

A similar assumption was made by professionals in relation to the ability to develop meaningful and supportive relationships through telehealth. Through wanting to provide a service that underpins traditional values of face-to-face care, professionals are potentially limiting access to services that patients experience as empowering, supportive and meaningful.

Interestingly, both professionals and patients held preconceived ideas about who would be suitable for telehealth services, identifying younger individuals as likely to be more accepting and identifying older adults as being more likely to struggle with adopting new technologies. This preconception was challenged by the lived experience of the patients themselves, who described developing mastery of the equipment despite initial anxieties and challenges with the technology.

In summary, the findings raise interesting questions about the important gatekeeper role that professionals assume when selecting services for their patients. Professionals appear to negotiate a fine line between consideration of their patients' needs through an attempt to place themselves in their position, whilst inadvertently and often subconsciously, attempting to satisfy their own psychological needs for autonomy, competence and relatedness whilst fulfilling their occupational role. In doing so, it appears that for some professionals assuming an accurate patient perspective is difficult and that patients are often seen within the context of their condition and less in the context of their broader lives.
Whilst patient-centred decision-making is advocated and adopted by most professionals, this becomes diluted when patients are not afforded the opportunity to access services such as telehealth either because of preconceived ideas about whether they would want to access care this way.

However, this tension raises broader questions about whether, despite providing potential benefits and psychological need satisfaction for patients; telehealth inadvertently thwarts psychological needs for the professionals working with technology and who maintain a preference for a face to face, traditional model of service provision with regular human contact.

Overall, telehealth appeared to support satisfaction of autonomy, competence, and relatedness needs of patients. However, professionals perceived more opportunities for telehealth to thwart patient needs than satisfy them. Professionals framed their perspectives through the lens of a more biomedical model of care as opposed to having a full biopsychosocial perspective of the environmental factors outside of the clinical context that influence the satisfaction of patients psychological needs.

Current policy suggests that effort should be made to ensure care can be provided within a community setting, and that patients should take an active role in their health care decisions [5, 6]. A greater awareness of this divergent reasoning may be necessary to enable telehealth to become a potential tool to meet these objectives.

Acknowledgements The authors would like to acknowledge thanks to Gudrun Jones, Hywel Dda University Health Board for her contribution to delivering the psychotherapy sessions as part of this project and to the participants who took part. Thanks, are also extended to Carys Stevens, Hywel Dda UHB for her clinical support in organizing the research.

Funding This research was funded by the Knowledge Economy Skills Scholarship (KESS). KESS is a pan-Wales higher level skills initiative led by Bangor University on behalf of the HE sector in Wales. It is part-funded by the Welsh Government's European Social Fund (ESF) convergence program for West-Wales and the Valleys.

Data Availability Unfortunately, the authors did not ask participants to provide consent for their data to be stored in an openly accessible online repository and therefore research data for this study is not shared. However, the authors invite any and all questions regarding data, which they will provide as much information as possible covered by the consent provided by participants already.

\section{Declarations}

Ethical approval Ethical approval was received by the National Health Services Research Ethics Committee (12/WA/0398). The study was conducted in accordance with the Declaration of Helsinki.

Consent to participate All participants gave fully informed consent to participate in this study before taking part in any research activities. 
Consent to publication All participants provided their consent for anonymised quotes to be used in the publication of the results of the study.

Conflict of interest The authors declare that they have no conflict of interest.

Open Access This article is licensed under a Creative Commons Attribution 4.0 International License, which permits use, sharing, adaptation, distribution and reproduction in any medium or format, as long as you give appropriate credit to the original author(s) and the source, provide a link to the Creative Commons licence, and indicate if changes were made. The images or other third party material in this article are included in the article's Creative Commons licence, unless indicated otherwise in a credit line to the material. If material is not included in the article's Creative Commons licence and your intended use is not permitted by statutory regulation or exceeds the permitted use, you will need to obtain permission directly from the copyright holder. To view a copy of this licence, visit http://creativecommons.org/licenses/by/4.0/.

\section{References:}

1. Koch S. Home telehealth-current state and future trends. International journal of medical informatics. 2006. https://doi. org/10.1016/j.ijmedinf.2005.09.002.2005.09.002

2. Robinson CA, Pesut B, Bottorff JL. Issues in rural palliative care: views from the countryside. J Rural Health. 2010. https://doi.org/l 10.1111/j.1748-0361.2009.00268.x.

3. Department of Health and Social Care. End of Life Care Strategy: Promoting high quality care for adults at the end of life. 2008. www. gov.uk/government/publications/end-of-life-care-strategypromoting-high-quality-care-for-adults-at-the-end-of-their-life. Accessed 11th Jan 2021.

4. Longley M, Llewellyn M, Beddow T. Mid-Wales Healthcare Study - Report for Welsh Government. 2014. www. midwalesjointcommittee.wales.nhs.uk/sitesplus/documents/1158/ MWHS_Report_-_WIHSC_for_Welsh_Government.pdf . Accessed 11th Jan 2021.

5. Whitten P, Doolittle G, Mackert M. Telehospice in Michigan: use and patient acceptance. American Journal of Hospice and Palliative Medicine®. 2014. https://doi.org/10.1177/104990910402100307.

6. Becevic M, Boren S, Mutrux R, Shah Z, Banerjee S. User Satisfaction With Telehealth: Study of Patients, Providers, and Coordinators. Health Care Manag. 2015. https://doi.org/10.1097/ HCM.0000000000000081.

7. Bush N, Donaldson G, Moinpour C, Haberman M, Milliken D, Markle V, Lauson J. Development, feasibility and compliance of a web-based system for very frequent QOL and symptom home selfassessment after hematopoietic stem cell transplantation. Qual Life Res. 2005; 14:77-93.

8. Whitten P, Holtz B, Meyer E, Nazione S. Telehospice: reasons for slow adoption in home hospice care. J Telemed Telecare. 2009. https://doi.org/10.1258/jtt.2009.080911.

9. Nicholson J, Coates L, Mountain G, Hawley M. Barriers and facilitators to mainstreaming telehealth in the communityexploring staff views and roles at the implementation and delivery phase. International Journal of Integrated Care. 2013. https://doi. org/10.5334/ijic.1417.
10. Mair FS, Hiscock J, Beaton SC. Understanding factors that inhibit or promote the utilization of telecare in chronic lung disease. Chronic Illness. 2008. https://doi.org/10.1177/1742395308092482.

11. May C. A rational model for assessing and evaluating complex interventions in health care. BMC Health Serv Res. 2006. https:// doi.org/10.1186/1472-6963-6-86.

12. Deci E, Ryan RM. Intrinsic motivation and self-determination in human behavior: Springer Science \& Business Media. 1985. https:// doi.org/10.1007/978-1-4899-2271-7_2.

13. Deci EL, Ryan RM. The, "what" and "why" of goal pursuits: Human needs and the self-determination of behavior. Psychol Inq. 2000. https://doi.org/10.1207/S15327965pli1104_01.

14. Spradley JP. The ethnographic interview. Belmont, CA: Wadsworth; 1979.

15. Hsieh HF, Shannon SE. Three approaches to qualitative content analysis. Qual Health Res. 2005. https://doi.org/10.1177/ 1049732305276687.

16. Elo S, Kyngas H. The qualitative content analysis process. J Adv Nurs. 2008. https://doi.org/10.1111/j.1365-2648.2007.04569.x.

17. Braun V, Clarke V. Using thematic analysis in psychology. Qualitative research in psychology. 2006. https://doi. org/10.1191/1478088706qp063oa.

18. Reeve J, Jang H, Hardre P, Omura M. Providing a rationale in an autonomy-supportive way as a strategy to motivate others during an uninteresting activity. Motivation and emotion. 2002. https:// doi.org/10.1023/A:1021711629417.

19. Venkatesh V, Morris MG, Davis GB, Davis FD. User acceptance of information technology: Toward a unified view. MIS quarterly. 2003. https://doi.org/10.2307/30036540.

20. Radhakrishnan K, Jacelon C, Roche J. Perceptions on the use of telehealth by homecare nurses and patients with heart failure: A mixed method study. Home Health Care Management \& Practice. 2012. https://doi.org/10.1177/1084822311428335.

21. May CR, Mair F, Finch T, MacFarlane A, Dowrick C, Treweek S, Montori VM. Development of a theory of implementation and integration: Normalization Process Theory. Implement Sci. 2009. https://doi.org/10.1186/1748-5908-4-29.

22. Ludwig-Beymer, P. Creating culturally competent organizations. Transcultural Concepts in Nursing Care. 4th ed. Philadelphia: Lippincott Williams \& Wilkins; 2003.

23. Demiris G, Oliver DP, Courtney KL. Ethical considerations for the utilization of telehealth technologies in home and hospice care by the nursing profession. Nursing Administration Quarterly. 2006. https://doi.org/10.1097/00006216-200601000-00009.

24. Sabesan S, Allen D, Caldwell P, Loh P, Mozer R, Komesaroff PA, Grabinski O. Practical aspects of telehealth: doctor-patient relationship and communication. Internal medicine journal. 2014. https://doi.org/10.1111/imj.12323.

25. Dalley D, Rahman R, Ivaldi A. Health Care Professionals' and Patients' Management of the Interactional Practices in Telemedicine Videoconferencing: A Conversation Analytic and Discursive Systematic Review. Qualitative Health Research. 2020. https://doi.org/10.1177/1049732320942346.

26. Schmidt KL, Gentry A, Monin JK, Courtney KL. Demonstration of Facial Communication of Emotion Through Telehospice Videophone Contact. Telemedicine and E-Health. 2011. https://doi. org/10.1089/tmj.2010.0190. 